\title{
Introduction to Topics in Modelling Financial and Macroeconomic Time Series
}

\author{
Fredj Jawadi ${ }^{1}$ \\ Published online: 1 July 2020 \\ (c) Springer Science+Business Media, LLC, part of Springer Nature 2020
}

\begin{abstract}
This special issue of Computational Economics features recent and original studies, most of which were presented at the fourth International Workshop in Financial Markets and Nonlinear Dynamics (FMND), organized in Paris in June 2019 (www. fmnd.fr). The papers describe and apply recent developments in financial econometrics to model the properties of financial and macroeconomic data. They present different methodologies and provide interesting and original findings.
\end{abstract}

Keywords Time Series modelling $\cdot$ Financial data $\cdot$ Macroeconomic data

\section{JEL Classifications C22 · G15}

Over the last few decades, there has been a growing body of literature on modelling financial and macroeconomic data, facilitated by easy access to a considerable amount of data as well as the availability of sophisticated machines which have helped scholars to datamine a large quantity of information. Further, since the aftermath of the global financial crisis in 2008-2009, many of the financial and macroeconomic models used in the past have been subject to criticism for yielding different back-testing results and extensions.

The current special issue of Computational Economics has published a series of twelve original research papers, presented at the fourth international workshop of financial markets and nonlinear dynamics organized in Paris in June 2019 (www. fmnd.fr). We briefly introduce and present these papers, grouping them by field of research.

We begin with two papers in the domain of portfolios. The first paper, by Detao Zhang and his co-authors, focuses on the selection of an optimal portfolio for investors under uncertain exit random time, with a focus on information costs and short

Fredj Jawadi

fredj.jawadi@univ-lille.fr

1 University of Lille, Office B655, 104 Avenue du Peuple Belge, 59043 Lille Cedex, France 
sales constraints. Maximizing the ratio between the wealth and the value of fixed assets, the authors develop an optimal portfolio choice strategy and empirically validate it through simulations. Jean-Luc Prigent and his co-authors also determine an optimal financial portfolio under the assumption of ambiguity aversion. In particular, they adopt a framework that enables investors to invest in various risky assets. Formally, they investigate the CRRA framework, while introducing ambiguity using the entropy criterion. Accordingly, the authors show that ambiguity regarding the correlation between risky assets might impact the optimal payoff.

Modelling connectedness is the focus of the next two papers in this special issue. Paper 3 by Philip Franses applies non-parametric Dynamic Time Warping (DTW) to study similarities across economic time series. The author shows that DTW is able to capture alternations between lead-lag series relations. Through an application to US data, the author highlights further evidence of temporal alignments across state dynamics of the US business cycle around the great recession. He also shows state-varying recoveries from the recession. In paper 4 by Apostolos Serletis and his co-authors, the authors address the estimation of singular demand systems under the assumption of a time-varying matrix of errors for the demand system, while considering the constant conditional correlation (CCC) and dynamic conditional correlation (DCC) parameterizations of the variance model. The authors derive a number of important practical results and also provide an empirical application to support their methodology.

The next three papers in this special issue highlight the interest of using timevariation modelling. Paper 5, by Juan Sapena and his co-authors, develops a flexible state-space framework to model time series data to develop a mean-reverting panel time-series model. This econometric framework is particularly useful to capture further asymmetry and nonlinearity in the data. Applying the Feldstein-Horioka puzzle in a 17-country panel, the authors show the interest of their model, especially for solving complexities within macroeconomic data. Stephen Hall and his co-authors carried out a series of Monte Carlo Simulations to examine the performance of a time-varying-coefficient (TVC) procedure (paper 6) that deals with measurement errors, omitted variables, incorrect functional forms and simultaneity. In particular, the authors developed a new Bayesian search technique to set the appropriate driver variables for this TVC methodology. Paper 7 by Nabila Jawadi and her co-authors demonstrates the interest of time-varying modelling to compute the effects of investor attention on Islamic Stock returns. In particular, it appears that investor attention might drive investment is Islamic stock bonds, but this effect is time-varying and changes with the quantile under consideration.

The next three papers develop specific technical procedures that aim to optimize the datamining of financial time series. In particular, paper 8 by R. Neck develops a new OPTCON 3 algorithm to determine optimal policies for stochastic control problems with a quadratic objective function and nonlinear dynamic models. While including active learning and the dual effects of optimizing policies, the author points to the interest of an OPTCON3 approach to enhance our understanding of the adaptive economic policy problem under uncertainty. Carlos Rivero is the author of paper 9 which proposes a new procedure for Constant Parameters in a stochastic volatility model. In particular, the author proposes a new way to test the constancy of 
parameters in the stochastic volatility equation. He demonstrates the relevance and performance of his method using a Monte Carlo simulation. Paper 10 by Chengzhao Zhang introduces the kernel method into fuzzy c-mean algorithm (FCM) and synthetic minority over-sampling technique (SMOTE), combining them with a support vector machine (SVM) to propose a hybrid model of KFCM-KSMOTE-SVM for the Chinese stock market (China Securities Index 300). Empirically, the author shows that the KFCM-KSMOTE-SVM significantly outperforms various other prediction models, suggesting that KFCM-KSMOTE-SVM can solve the class imbalance problem in financial markets and predict extreme financial risks.

Finally, the two last papers in this issue deal with fascinating topics in banking and asset pricing. In paper 11, Sushanta Mallick and his co-author take bank ownership structures into consideration, while checking for improvements to efficiency in the convergence of banks in India during the post-reform period. Applying a nonparametric frontier estimator's approach to a balanced panel of 73 banks, the authors compute time-dependent bank efficiency scores in order to examine the dynamics of the technological frontier and catch-up levels of Indian banks, pointing to the superiority of state-owned banks in India. In the last paper, by Jean-Luc Prigent and his co-author, the pricing process of a Bermuda swaption is explained from a computational point of view, and the process that provides a Bermuda swaption price is developed. Accordingly, using a 3-factor hybrid model based on the Libor market model that combines the domestic market, the foreign market and the foreign exchange market, they find further evidence of significant relationships between the cross-currency product and the volatility of each of the three markets under consideration.

Publisher's Note Springer Nature remains neutral with regard to jurisdictional claims in published maps and institutional affiliations. 